\title{
Erratum to: Erythrobacter westpacificensis sp. nov., a Marine Bacterium Isolated from the Western Pacific
}

\author{
Jingjing Wei $\cdot$ Yujiao Mao $\cdot$ Qiang Zheng $\cdot$ \\ Rui Zhang $\cdot$ Ya-Nan Wang $\cdot$ Nianzhi Jiao
}

Published online: 11 January 2013

(C) Springer Science+Business Media New York 2013

\section{Erratum to: Curr Microbiol}

DOI 10.1007/s00284-012-0287-0

The original version of this article unfortunately contained few mistakes. Here are the corrections for it.

1. The name of a co-author, Nianzhi Jiao is missing in the original published version.
The correct co-authors are as follows:

Jingjing Wei · Yujiao Mao · Qiang Zheng · Rui Zhang · Ya-Nan Wang · Nianzhi Jiao

2. The corresponding author name in the original published version is wrong.

The correct corresponding author name is Nianzhi Jiao

The online version of the original article can be found under doi:10.1007/s00284-012-0287-0.

J. Wei · Y. Mao - Q. Zheng · R. Zhang · Y.-N. Wang .

N. Jiao $(\square)$

State Key Laboratory for Marine Environmental Science,

Institute of Marine Microbes and Ecospheres, Xiamen

University, Xiamen 361005, People's Republic of China

e-mail: jiao@xmu.edu.cn

J. Wei

e-mail: weijingjingxmu2012@gmail.com

Y.-N. Wang

Key Laboratory of Microbial Engineering at the Institute of Biology, Henan Academy of Sciences, Zhengzhou 450008,

People's Republic of China 